\title{
The first uncooled (no thermal) MWIR FPA monolithically integrated with a Si-CMOS ROIC: a 80x80 VPD PbSe FPA
}

\author{
G. Vergara, R. Linares-Herrero, R. Gutiérrez-Álvarez, C. Fernández-Montojo, L.J. Gómez, V. \\ Villamayor, A. Baldasano-Ramírez and M.T. Montojo. \\ New Infrared Technologies S.L., Vidrieros 30, Boadilla del Monte, Madrid-28660, Spain
}

\begin{abstract}
In this work a breakthrough in the field of low-cost uncooled infrared detectors is presented: an $80 \times 80$ FPA of VPD PbSe with MWIR detection in uncooled operation. The new device represents a milestone in the road towards affordable uncooled MWIR imagers, once the Vapor Phase Deposition $\mathrm{PbSe}$ technology (aka VPD PbSe) has reached the industrial maturity. The devices presented in this work, in which the active material has been monolithically integrated with the corresponding Si-CMOS readout circuitry (ROIC), were processed on $200-\mathrm{mm}$ Si wafers with excellent results in terms of manufacturing yield and repeatability. Fast speeds of response and high frame rates were, until date, non-existing performances in the low-cost uncooled IR imagers domain: this new detector fills the gap, being capable to provide MWIR images with rates as high as $2 \mathrm{KHz}$, full frame, snapshot imaging and real uncooled operation. The technology opens the MWIR band to SWaP concept.
\end{abstract}

Key words: Uncooled MWIR FPA, high speed, monolithic integration, PbSe.

\section{Introduction}

Nowadays, the term 'uncooled infrared detection' is synonymous of thermal detectors. It is possible to find uncooled thermal IR imagers with performance/cost ratios never imagined a few years ago, causing that the number of new applications has grown exponentially as a result of the continuous and significant cost reduction of uncooled imagers experimented during the last years. This is causing that the IR detector markets are moving towards unexplored fields of applications with plenty of new opportunities and challenges.

In this scenario, new and more demanding needs appears every day. Fast speeds of response, high frame rates and/or good sensitivity in the MWIR (1-5 microns) spectral band in uncooled operation are frequent industrial and defense needs which are waiting for a new type of uncooled IR detectors rather than thermal, which performance in terms of spectral band of response, velocity of response and sensitivity, is limited by fundamental reasons.

Polycrystalline lead salts have excellent performances in uncooled operation but their technologies have been stigmatized during a long time for limitations in material processing and a poorly understood physics. During the last years a new way of processing $\mathrm{PbSe}$ infrared detectors based on a Vapor Phase Deposition (VPD) method [1] has opened new and excellent prospectives for filling the gap existing in the field of quantum uncooled MWIR imaging devices. The new method to process the detectors is based on a deposition of a thin film of $\mathrm{PbSe}$ on a substrate by thermal evaporation, followed by a specific high temperature thermal treatment to make the $\mathrm{PbSe}$ to the IR radiation.

The advantages of using the VPD method versus the classic, standard process (Chemical Bath Deposition aka CBD) for PbSe processing are directly related with the possibility offered by the VPD method of using big area and complex substrates.

VPD PbSe technology is unique as it combines all the main requirements demanded for a volume ready technology:

1. Affordable processing

2. Good reproducibility and homogeneity

3. Processing compatible with big area substrates

4. Si-CMOS compatible (no hybridization needed)

5. Mature

6. Compatible with low cost packaging (no need of vacuum) 


\section{Low cost optics (1-5 microns).}

In 2007 the compatibility of standard Si-CMOS circuitry and the VPD PbSe technology was demostrated. The first device processed directly on a CMOS-ROIC was a prototype of FPA with $16 \times 16$ DPS pixel type [2]. In this work it is presented the first uncooled (no thermal) 2D FPA monolithically integrated with their corresponding Si-CMOS ROIC fabricated using industrial ready processes. It is a prototype of an $80 \times 80$ pixel VPD PbSe FPA processed on a 200-mm Si wafer. It is the first infrared quantum device fully compatible with Si-CMOS technology and could be considered a milestone for the next generation of low cost, high speed infrared imagers for industrial and/or defense applications.

Section 1 is focused on the Digital Pixel Sensor (DPS) pixel architecture. In section 2 some details of device processing are described, in section 3 the main characteristics and preliminary testing results of the processed devices are presented, whereas section 4 shows some conclusions of the work done.

\section{Pixel architecture}

The FPA format is a square of $80 \times 80$ pixels DPS (Digital Pixel Sensor) type. Each pixel is a complex cell with the following functionalities:

- Dark current cancellation

- Compensation of parasitic input capacitance

- Sensor effective current integration

- ADC

- Individual gain setting

- Internal analog bias generation

- Digital input and output

Pixel size is $130 \times 130 \mu \mathrm{m}^{2}$ and the CMOS technology used for ROIC processing was 4 metals / $0.35 \mu \mathrm{m}$. Figure 1 below shows a scheme of the DPS architecture.

Figure 2 shows pixel layout with the distribution of blocks in the pixel and their respective sizes.

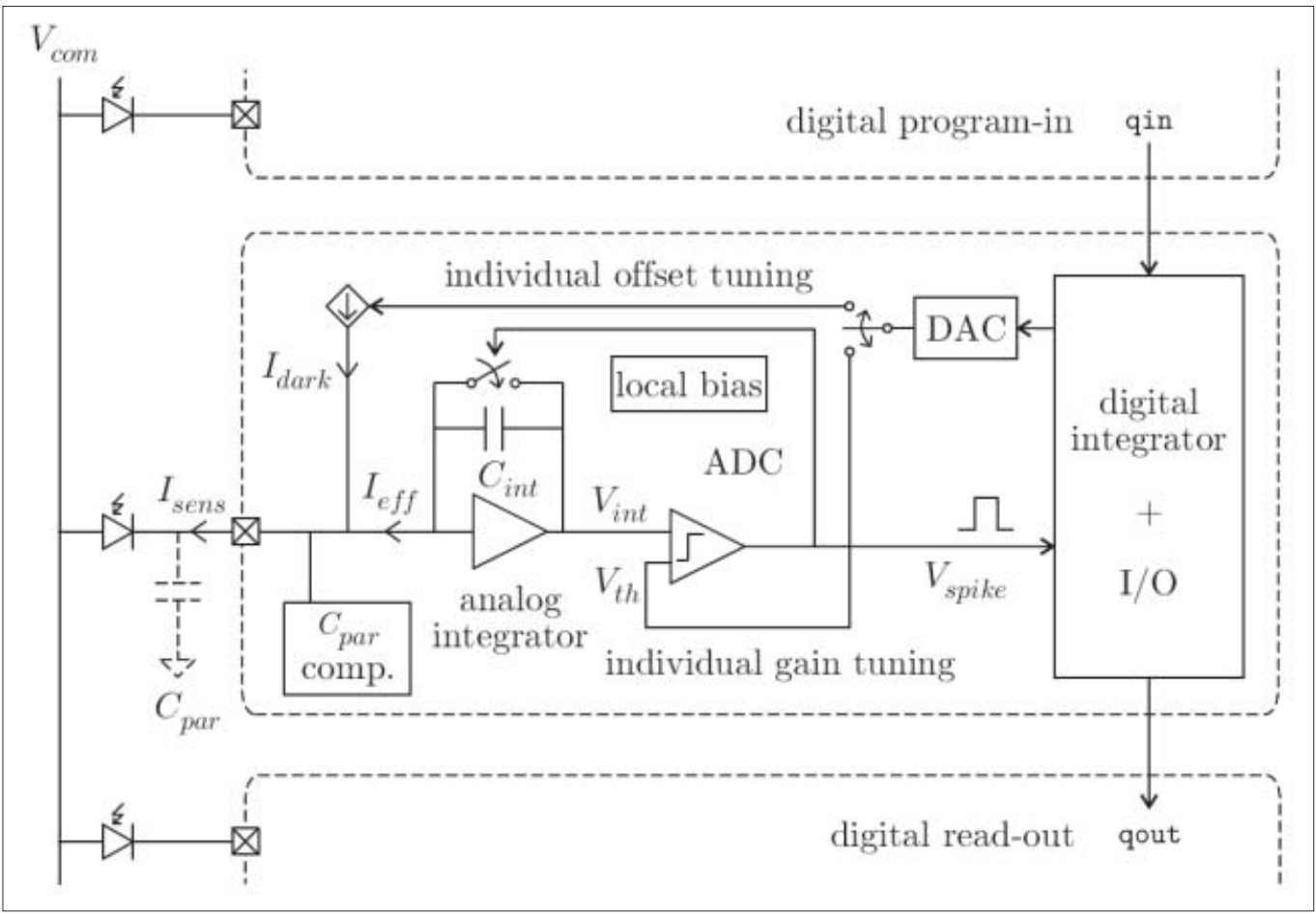

Fig. 1. Digital Pixel Sensor scheme 


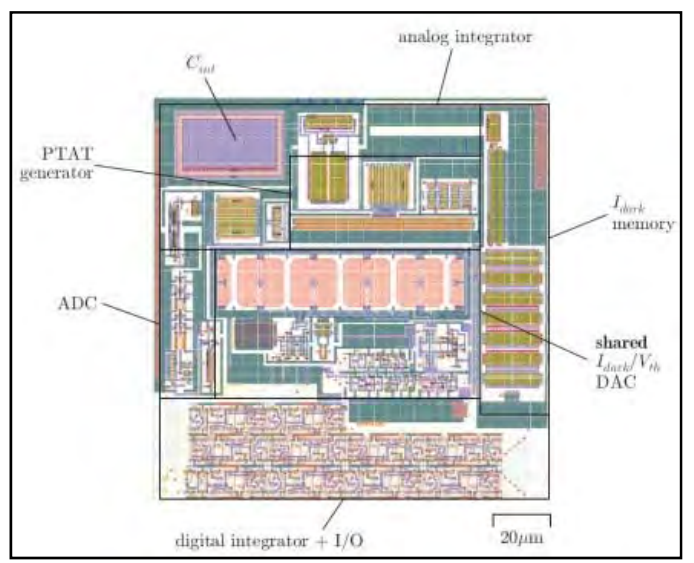

Fig. 2. Pixel blocks distribution

As a remarkable fact, the dark current cancelation and gain control are set using an external programming method based on the multiplexing of a common DAC inside the pixel electronics. The transistors in charge of canceling the dark current are dimensioned for providing a wide range of current values. Dark current and gain are programmed sequentially when writing each image. In this way, the hold time of the analog memory is shorter, allowing a reduction of the leak crosstalk and therefore the possibility of using external algorithms for dark current compensation in real time.

\section{Device processing}

Without any doubt the main advantage of the new device presented in this work, and the reason why it can be considered a breakthrough in the domain of uncooled infrared imagers, is that its fabrication is the result of having done fully compatible both, VPD PbSe and Si-CMOS technologies.

During development phase a variety of processes were modified and adapted to bigger substrates in order to improve technology feasibility and reliability. The efforts were applied in four main directions [3]:

a) Optimization of metal stacks for contacting electronics and active material: Si-CMOS standard technology is not compatible with metals used in the standard PbSe technology. It has been necessary to work on specific metal stacks for making both technologies fully compatible.

b) Adaptation of PbSe sensitization procedure to standard Si-CMOS technology requirements: after deposition, the VPD PbSe is not sensitive to the infrared radiation. It is necessary a specific high-temperature thermal treatment in order to convert it into an active material sensitive to the IR light. It was necessary to reduce the original temperature treatment profiles to levels below the standard Si-CMOS technology tolerances for keeping the CMOS circuits alive during $\mathrm{PbSe}$ sensitization processes.

c) Improving VPD deposition method in large area substrates: The PbSe deposited by VPD was adapted to wafers of $200-\mathrm{mm}$ of diameter. The method developed is scalable to bigger wafer diameters with minor modifications.

Once the ROICs are fabricated on $200-\mathrm{mm} \mathrm{Si}$ wafers using standard CMOS processes, the substrates are postprocessed in an easy way, depositing and patterning specific stacks of metals with the objective of improving the electrical contact between CMOS circuitry and VPD PbSe.

Then, the active material, $\mathrm{PbSe}$, is deposited by thermal evaporation in vacuum following a proprietary method. After PbSe deposition and patterning, the wafer is submitted to a specific thermal treatment for converting the PbSe into an infrared sensitive material.

Figure 3 shows the detail of a $200-\mathrm{mm}$ wafer fully processed with $32 \times 32$ (small squares) and $80 \times 80$ (big squares) FPAs of VPD PbSe.

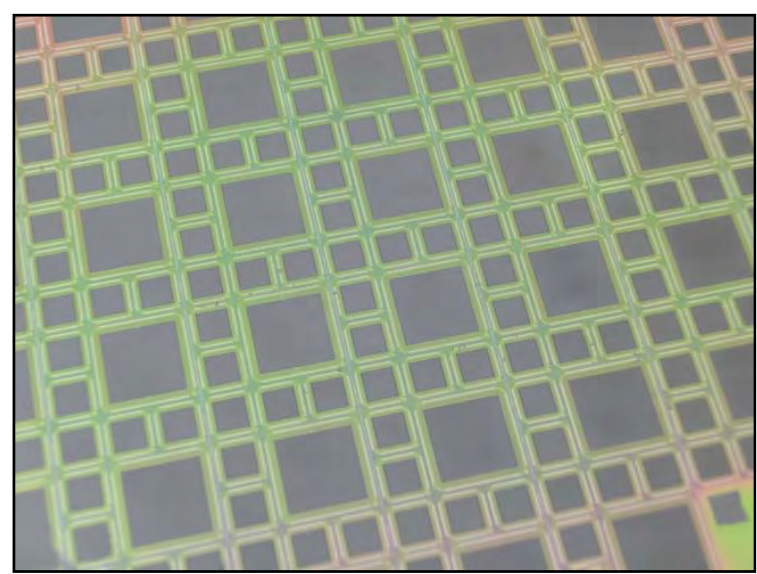

Fig. 3. Detail of a $200 \mathrm{~mm}$ substrate with $32 \times 32$ and $80 \times 80$ VPD PbSe FPAs processed on it.

After processing, the arrays are electro-optically tested on-wafer.

d) Low cost device packaging: As a feature associated with the quantum nature of the active material, the VPD Pbse detectors do not need to be packaged in vacuum. It can be considered an enormous advantage in terms of cost reduction, production yield and throughput. Packaging is a costly task in optoelectronics and represents a key step in the fabrication of infrared detectors: more than $60 \%$ of an 
infrared FPA cost is directly related with the packaging of the device.

Robustness and good performances, even working at atmospheric pressure, facilitate and simplify detector packaging. A packaging concept based on "on wafer" strategy was developed. The packaging method consists in covering the active area with an infrared window directly glued on the silicon surface. It is compatible with different window materials such as sapphire, silicon, chalcogenide glass etc. The packaging method developed has the advantage of unifying flexibility for packaging different device sizes and geometries, wide area substrates and low cost. After being covered with a transparent window the detectors are separated, assembled on a PCB, wire bonded and dam and filled.

In figure 4 it is possible to observe in detail a few of the processed devices on a Si-wafer covered with sapphire windows before being diced and separated.

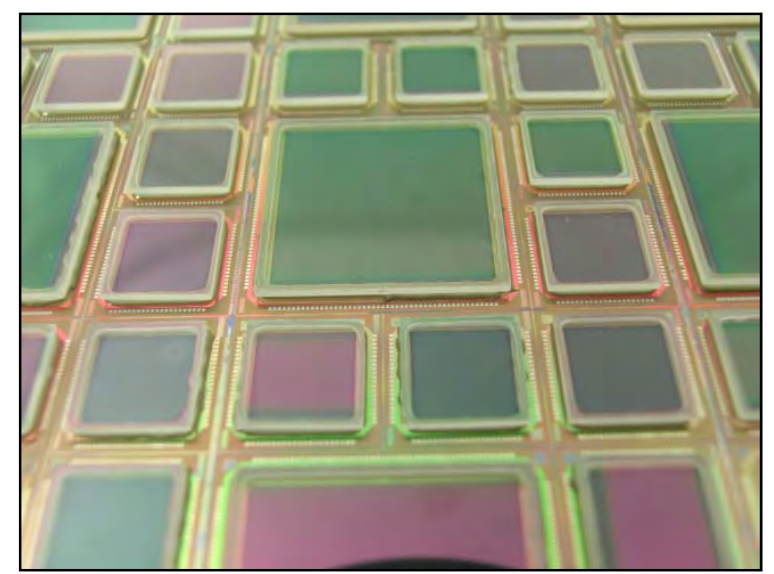

Fig. 4. Detail of a $200 \mathrm{~mm}$ Si substrate with $32 \times 32$ and $80 \times 80$ VPD PbSe FPAs covered with sapphire windows.

\section{Device characteristics and testing}

Before dicing, every FPA on the wafer surface is submitted to a preliminary "on wafer" characterization using a probe card and a specific software designed for programming and reading sequentially each pixel of the FPA. Figure 5 shows the experimental set up used for testing each device processed on the surface of a wafer. Functional tests and preliminary electrooptical measurements allow to select and classify the devices according to several criteria such as uniformity, sensitivity among others.

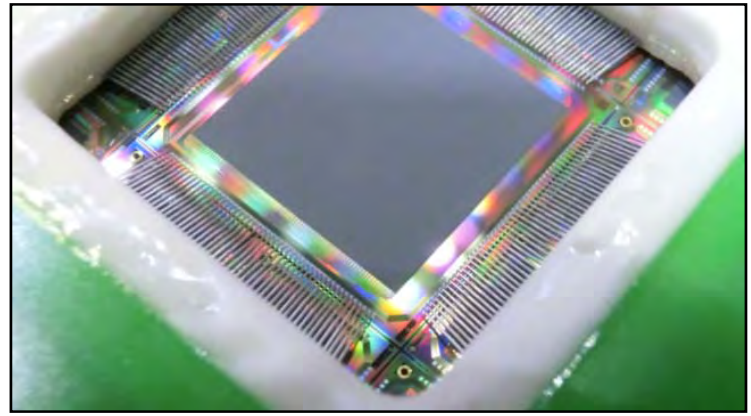

Fig. 5. Functional on wafer testing of a $80 \times 80$ VPD PbSe FPA

Once separated, the FPAs are mounted, wire bonded and filled directly on PCB using the low cost, volume suitable packaging method described before. Figure 6 shows an $80 \times 80$ FPA mounted on a specific testing PCB.

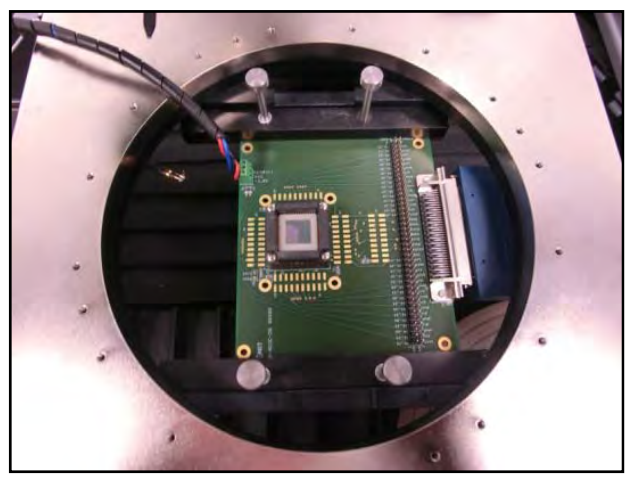

Fig. 6. A $80 \times 80$ VPD PbSe FPA on testing PCB

The devices are then electrooptically characterized. The $80 \times 80$ FPA obtained show the following characteristics summarized below:

- FPA resolution: 80x80 (6400 pixels)

- Pixel size: 130 x 130 um2 (square format)

- Pixel pitch: 135 um

- $A / D$ readout electronics: on-chip

- Dark current cancellation: on-chip

- Digital interface for FPA control and data acquisition/transmission

- Readout method: Snapshot

- Data format: raw, 10 bit (depth)

- Packaging: SMD / LCC 64 pins $(4 \times 16)$ / $720 \times 720$ mils / pitch: 40 mils

- Power supply $(3.3 \mathrm{~V},<0.5 \mathrm{~W}$ ) (digital \& analog)

- Detector biasing voltage: $-5 \mathrm{~V}$ 
- Control: 5 lines

- Gain, offset control (per pixel): 16 lines (serial communication, $10 \mathrm{MHz}$ )

- Data output: 16 lines (serial communication, $10 \mathrm{MHz}$ )

- Readout speed: > 2000 frames per second

- Integration time: programmable, 100 us - 1 $\mathrm{ms}$

An intensive electrooptical characterization process of a complete batch of devices is being carried out at the publication time of this work. The preliminary results obtained are very promising in terms of device performance, yield and fabrication feasibility. Figure 7 shows an image obtained with an $80 \times 80$ VDP PbSe FPA. The image corresponds to a flame obtained to a frame rate above 2700 frames per second $(\mathrm{Hz})$.
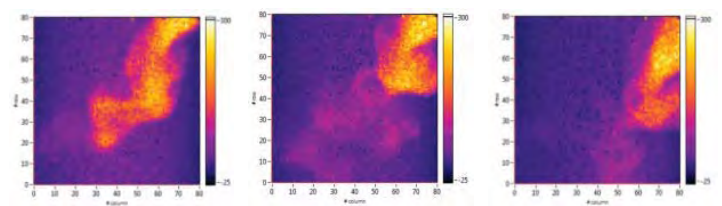

Fig. 7. Sequence of the temporal evolution of a flame. They are raw images obtained with a $80 \times 80$ real uncooled VPD PbSe FPA working @ 2.740 fps

\section{Conclusions}

The innovation presented in this work can be considered a milestone in the infrared imagers' domain. Even though it is a low resolution device $(80 \times 80$ pixels) it can be considered a breakthrough because it is the first uncooled photonic (no thermal) detector monolithically integrated with Si-CMOS technology.

The new uncooled FPA offers an unique and very innovative device where excellent performances, such as uncooled operation, low cost, high frame rate and a good sensitivity in the MWIR spectral region converge; it has been processed using a technology suitable for volume applications. There are not any existing solutions able to combine all these characteristics in the same detector. The new technology opens the MWIR spectral band to the SWaP (small Size, Weight and Power) concept.

\section{References}

[1] G. Vergara, F.J. Sánchez , M.T. Rodrigo, J. Diezhandino, M. ÁLvarez, M.C. Torquemada, V. Villamayor, M. Álvarez, J. Plaza, I. Catalán, R. Almazán, M. Verdú, P. Rodríguez, L.J. Gómez and M.T. Montojo, Polycrystaline lead selenide. The resurgence of an old infrared detector, Optoelectronics Review 15(2), 110-117 (2007)

[2] M.T. Montojo, G. Vergara F.J. Sánchez, M.T. Rodrigo, R. Gutierrez, M. Álvarez, M.C. Torquemada, V. Villamayor, J. Plaza, I. Catalán, R. Almazán, M. Verdú, P. Rodríguez and L.J. Gómez, Monolithic uncooled IR detectors of polycrystalline $\mathrm{PbSe}$. $A$ real alternative Proceedings SPIE 6542 (65), (2007).

[3] R. Linares-Herrero, M.T. Montojo, Arturo Baldasano-Ramírez, VPD PbSe technology the road towards the industrial maturity, Proc. of SPIE 7660 (2010) doi: 10.1117/12.850174. 